\title{
Effect of Intrauterine Administration of Seminal Plasma for Patients with Recurrent Implantation Failure Before FET: Study Protocol for a Prospective Randomised Controlled Trial
}

\section{ling CUI}

Chengdu Women's and Children's Central Hospital: Chengdu Women and Children's Central Hospital Fang Wang ( $\triangle$ postwf@163.com )

Chengdu Women's and Children's Central Hospital: Chengdu Women and Children's Central Hospital https://orcid.org/0000-0001-9543-7675

Yonghong Lin

Chengdu Women's and Children's Central Hospital: Chengdu Women and Children's Central Hospital

\section{Research Article}

Keywords: Recurrent implantation failure, Randomized controlled trial, clinical pregnancy rate, seminal plasma, frozen-thawed embryo transfer

Posted Date: December 29th, 2021

DOl: https://doi.org/10.21203/rs.3.rs-643898/v1

License: (c) (i) This work is licensed under a Creative Commons Attribution 4.0 International License.

Read Full License 


\section{Trials structured Study Protocol}

\section{Title}

Effect of intrauterine administration of seminal plasma for patients with recurrent implantation failure before FET: study protocol for a prospective randomised controlled trial

\section{Names protocol contributors}

Ling Cui, Yonghong Lin, Fang Wang

Department of Reproduction and Infertility, Chengdu Women's and Children's Central Hospital, School of Medicine, University of Electronic Science and Technology of China, Chengdu 611731, China.

Corresponding authors: Fang Wang, email: postwf@163.com

\section{Abstract}

- Background: To assess the effect of intrauterine administration of seminal plasma for patients with recurrent implantation failure before frozen-thawed embryo transfer.

Methods: Trial design: This is a parallel group, randomized (1:1 allocation ratio) controlled trial. Participants: All patients will be recruited from Chengdu Women's and Children's Central

Hospital.

Inclusion criteria: 1 . Women after IVF/ICSI treatment in Chengdu Women's and Children's Central Hospital. 2.Infertile women with a history of recurrent implantation failure. 3.Infertile couples (both male and female) aged between 20 and 39 years; 4 . Couples who have at least 1 goodquality embryos for transfer. 5. Males had negative in infectious disease test. 6 . The males have semen. 7. Women who intend to undergo FET after IVF or ICSI or pre-implantation genetic testing for aneuploidy (PGT-A). 8. Competent and able to give informed consent.

Intervention and comparator: Treatment group receiving intrauterine administration of seminal plasma before frozen-thawed embryo transfer.

Main outcomes: Clinical pregnancy after frozen-thawed embryo transfer.

Randomisation: Patients will be randomly allocated to either treatment or control group at 1:1 ratio. Random numbers will be generated by using software SPSS 25.0 performed by an independent statistician from Chengdu Women's and Children's Central Hospital.

Blinding (masking): Only the data analyst will be blinded to group assignment.

Numbers to be randomised (sample size): To account for a 10\% dropout rate, we will recruit 174 patients (87 in each group).

Trial status: The date of approval is 31 rd May 2021, version 2.0. Recruitment started on 1rd June and is expected to end in July 2022.

- Discussion: Intrauterine administration of seminal plasma before frozen-thawed embryo transfer of patients with recurrent implantation failure may improve clinical pregnancy rate, it has great 
significance for assisted reproduction.

Trial registration: The study protocol has been approved by the ethics committees at Chengdu Women's and Children's Central Hospital. The trial was registered at the Chinese Clinical Trial Registry ChiCTR2100046803. Registered on 28 May 2021.

\section{Keywords}

Recurrent implantation failure, Randomized controlled trial, clinical pregnancy rate, seminal plasma, frozen-thawed embryo transfer

\section{Administrative information}

Administrative information table:

Note: the numbers in curly brackets in this protocol refer to SPIRIT checklist item numbers. The order of the items has been modified to group similar items (see http://www.equator-network.org/reporting-

guidelines/spirit-2013-statement-defining-standard-protocol-items-for-clinical-trials/).

\begin{tabular}{|l|l|}
\hline Title $\{1\}$ & $\begin{array}{l}\text { Effect of intrauterine administration of seminal plasma for } \\
\text { patients with recurrent implantation failure before FET: study } \\
\text { protocol for a prospective randomised controlled trial }\end{array}$ \\
\hline Trial registration $\{2 \mathrm{a}$ and $2 \mathrm{~b}\}$. & $\begin{array}{l}\text { The study protocol has been approved by the ethics committees } \\
\text { at Chengdu Women's and Children's Central Hospital. The trial } \\
\text { was registered at the Chinese Clinical Trial Registry } \\
\text { ChiCTR2100046803. Registered on 28 May 2021. }\end{array}$ \\
\hline Protocol version $\{3\}$ & $\begin{array}{l}\text { The date of approval is 31rd May 2021, version 2.0. } \\
\text { Funding }\{4\}\end{array}$ \\
\hline Author details $\{5 \mathrm{a}\}$ & $\begin{array}{l}\text { This work was supported by the Sichuan Medical Association } \\
\text { grant (S18071) and Chengdu Women's and Children's Central } \\
\text { Hospital }\end{array}$ \\
\hline $\begin{array}{l}\text { Ling Cui, Yonghong Lin, Fang Wang } \\
\text { Department of Reproduction and Infertility, Chengdu Women's } \\
\text { and Children's Central Hospital, School of Medicine, } \\
\text { University of Electronic Science and Technology of China, } \\
\text { Chengdu 611731, China. } \\
\text { Corresponding authors: Fang Wang, email: postwf@ 163.com }\end{array}$ \\
\hline
\end{tabular}




\begin{tabular}{|l|l|}
\hline $\begin{array}{l}\text { Name and contact information for } \\
\text { the trial sponsor }\{5 b\}\end{array}$ & Ling Cui, tel. +8618181422637 , email: 306127383@ qq.com \\
\hline Role of sponsor $\{5 c\}$ & $\begin{array}{l}\text { Random numbers will be generated by using software SPSS } \\
\text { 25.0 performed by an independent statistician from Chengdu } \\
\text { Women's and Children's Central Hospital. The funding body } \\
\text { has no influence in the design of the study and data collection, } \\
\text { analysis and interpretation of data and in writing the } \\
\text { manuscript. }\end{array}$ \\
\hline
\end{tabular}

\section{Introduction}

\section{Background and rationale $\{6 a\}$}

Infertility is one of the most common problems among couples worldwide. In vitro fertilization (IVF) is widely used and well received in couples with reproductive difficulties, Since the first IVF baby was born in 1978. Recurrent implantation failure (RIF) after IVF/ intracytoplasmic sperm injection (ICSI) is a challenging topic for clinicians and can be a devastating reality for some patients with infertility.

RIF remains a variably defined condition, we reviewed the definition of RIF in recent studies[1-5], RIF may be defined as the repeated transfer of morphologically good embryos to a normal uterus without achieving successful implantation and a clinical pregnancy. The absence of implantation after three failed IVF or ICSI treatments, each with at least one fresh good quality embryo per transfer, or failure to achieve pregnancy after transfer of 10 good quality embryos[5,6]. It is different from recurrent IVF failure, because the main causes of recurrent IVF failure are low ovarian response and embryo quality, including RIF [3] .

Various uterine pathology including fibroids, endometrial polyps, congenital anomalies and intrauterine adhesions should be excluded by ultrasonography and hysteroscopy. Hydrosalpinges are a recognized cause of implantation failure and should be excluded by hysterosalpingogram; if necessary, laparoscopy should be performed to confirm or refute the diagnosis. Treatment offered should be evidence based, aimed at improving embryo quality or endometrial receptivity $[1,7,8]$ RIF is a source of pain and frustration for both patients and clinicians. In the absence of clinically useful testing, treatments are largely empirical and have limited efficacy. Successful embryo implantation is a dynamic process. The interaction between the embryo and the endometrium is influenced by the molecular interactions among cytokines, growth factors, hormones and cell adhesion molecules, and is the result of the interaction of multiple autocrine, paracrine and endocrine factors $[9,10]$.

Treatments of RIF are including immune therapy, anticoagulants, endometrial injury[11] and intrauterine perfusion of peripheral blood mononuclear cells (PBMC) treatments in recent years[12]. However, the effectiveness of these treatments is uncertain. Some treatments did not alter the clinical pregnancy rates, or live birth rates, such as endometrial scratching, evaluation of the 
endometrial receptivity assay, intrauterine granulocyte colony-stimulating factor (G-CSF) administration[13-15].

We are looking for more effective and direct treatments for RIF. Mating and/or sperm-free seminal plasma deposition in the reproductive tract after the expression of genes associated with spermlining epithelium interactions, ovulation, and pre-implantation effects. The seminal plasma can change the gene expressions reference to genes specifically associated with sperm-lining epithelium interactions (adhesion), ovulation, and pre-implantation (which can be separated from the free embryo influence) in the period $10-72 \mathrm{~h}$ post-mating[16]. Seminal plasma also induced the expression of the cytokines, granulocyte macrophage colony-stimulating factor, interleukin-6, etc. These changes were accompanied by altered dynamics in pre-implantation embryo development with an increase in the number of embryos and in their viability after seminal plasma treatment[17]. Experiments in rodents show seminal factor signalling acts to 'condition' the female immune response to tolerate the conceptus, and to organize molecular and cellular changes in the endometrium to facilitate embryo development and implantation[18].

Differ from mate, we don't need sperms in semen for patients with frozen-thawed embryo transfer (FET). The active factors in seminal plasma are higher in seminal plasma-sperm free after centrifugation.

\section{Objectives $\{7\}$}

The objective of this study is to analyze the effect of intrauterine administration of seminal plasma on clinical pregnancy rate in FET of patients with recurrent implantation failure. To verify whether the clinical pregnancy rate of patients with repeated embryo transfer can be improved, it has great significance for assisted reproduction.

\section{Trial design $\{8\}$}

This is a prospective, randomized, parallel-group, clinical trial to evaluate whether the intrauterine administration of seminal plasma could improve the clinical pregnant rate in FET cycles for patients with RIF. Eligible patients will be randomly assigned to the treatment group or control group with a $1: 1$ ratio.

\section{Methods: Participants, interventions and outcomes}

\section{Study setting $\{9\}$}

Chengdu Women's and Children's Central Hospital.

\section{Eligibility criteria $\{10\}$}




\section{Inclusion criteria}

1. Women after IVF/ICSI treatment in Chengdu Women's and Children's Central Hospital;

2. Infertile women with a history of RIF: RIF may be defined as the repeated transfer of morphologically good embryos to a normal uterus without achieving successful implantation and a clinical pregnancy.

a) Three or more embryo transfer cycles;

b) Or failure to achieve pregnancy after transfer of 4 good quality embryos, criteria of good-quality embryos are shown in Table 1);

3. Infertile couples (both male and female) aged between 20 and 39 years;

4. Couples who have at least 1 good-quality embryos for transfer;

5. Males had negative in infectious disease test;

6. The males have semen;

7. Women who intend to undergo FET after IVF or ICSI or pre-implantation genetic testing for aneuploidy (PGT-A);

8. Competent and able to give informed consent.

\section{Exclusion criteria}

1. Women with acute genital tract infection;

2. Women with severe mental illness;

3. Women with known autoimmune diseases;

4. Women with serious heart, brain and lung diseases and serious liver and kidney dysfunction;

5. Women diagnosed of endometritis without treatment

Gynecologist qualified for human assisted reproduction in China will perform the interventions.

\section{Who will take informed consent? $\{26 \mathrm{a}\}$}

The Informed Consent shall be explained in detail by the investigator to the participants and authorised surrogates. After the participants and authorised surrogates has fully read and understood the Informed Consent, if the they agrees to participate in the clinical trial, the informed consent shall be signed and dated by them. The researchers are also required to sign and date the informed consent. They will be told that their participation is entirely voluntary and that they can leave the trial at any time without their standard care being affected. 


\section{Additional consent provisions for collection and use of participant data and biological specimens $\{26 \mathrm{~b}\}$}

Not applicable.

\section{Interventions}

\section{Explanation for the choice of comparators $\{6 \mathrm{~b}\}$}

Choice of comparators. Routine preparation of endometrium without seminal injection before FET.

\section{Intervention description $\{11 a\}$}

The patients were randomized into two groups: treatment group receiving intrauterine administration of seminal plasma before ET and a control group undergoing ET without a previous intrauterine administration of seminal plasma.

Participants will undergo the frozen-thawed embryo transfer (FET) process (Table 1). Preparation of endometrium

For frozen/thawed embryo transfer, the receptivity of the endometrium was prepared by spontaneous menstrual cycle or hormone replacement therapy.

If the endometrium was $\geqslant 6 \mathrm{~mm}$, and the progesterone $(\mathrm{P})$ level was $\leqslant 1.5 \mathrm{ng} / \mathrm{mL}$, the frozenthawed ET was scheduled.

1) Transform endometrium: The progesterone ( $40 \mathrm{mg}$ i.m.,Xinan Pharmaceuticals, Chongqing, China) injection for a single daily administration started 3 or 5 days before ET.

Treatment group will receive seminal plasma via intrauterine insemination catheter before embryo transfer.

Control group will not receive intrauterine administration of seminal plasma before ET.

2) Embryo transfer

One or two blastocyst/cleavage embryos will be transferred in each participant.

At least one embryo transferred will be high-quality embryo. The serum hCG test is performed after 2 weeks of transfer. If the patient is pregnant, luteal phase support will be continued until 8-12 weeks of gestation and follow-up will be continued to the end of pregnancy.

The standard of high-quality embryo development rate is according to the Istanbul criteria[21] as following: Grade 1 - good rating: 10\% fragmentation, stage-specific cell size, no multinucleation. Grade 2 - fair rating: 10-25\% fragmentation, stage-specific cell size for majority of cells, no evidence of multinucleation. Grade 3 - poor rating: severe fragmentation ( $>25 \%)$, cell size not stage specific, evidence of multinucleation.

\section{Criteria for discontinuing or modifying allocated interventions $\{11 \mathrm{~b}\}$}


Reasons for discontinuation of treatment may include, but are not limited to, the following: (1) participants who become pregnant spontaneously, (2) participants who have experienced some complications or serious side effects), (3) participants who request to withdraw from the trial.

\section{Strategies to improve adherence to interventions $\{11 \mathrm{c}\}$}

Give the contact information of the project leader to the patient and keep in touch at any time.

\section{Relevant concomitant care permitted or prohibited during the trial $\{11 \mathrm{~d}\}$}

Medication for concomitant diseases is allowed. Such as thyroxine tablet for tryroid hypofunction, prednisone for dysimmunity. All other medicine including Chinese traditional medicine are prohibited during the trial.

\section{Provisions for post-trial care $\{30\}$}

Through telephone follow-up survey, and Chengdu Women's and Children's Central Hospital will be responsible for compensation to those who suffer harm from trial participation.

\section{Outcomes $\{12\}$}

\section{Primary outcome}

The primary outcome is clinical pregnancy after frozen-thawed embryo transfer, defined as a pregnancy diagnosed by ultrasonographic visualization of one or more gestational sacs or definitive clinical signs of pregnancy. It includes ectopic pregnancy[22]. Note: Multiple gestational sacs are counted as one clinical pregnancy.

\section{Secondary outcomes}

The secondary outcomes include biochemical pregnancy, pregnancy loss, implantation pregnancy rate, and perinatal complication and other adverse events. Biochemical pregnancy is defined as serum $\beta$-hCG $\geq 10 \mathrm{mIU} / \mathrm{mL}$ measured $12-15$ days after embryo transfer. Implantation rate is calculated as the number of gestational sacs by a transvaginal ultrasound scan/number of embryos transferred.

\section{Adverse events}

During the study, serious adverse events will be immediately reported to the principal investigator, and appropriate measures will be initiated instantly.

The Ethics Committee will determine whether the adverse event is likely to have been associated with the experimental drug, and the sponsor will provide the cost of the treatment associated with the study and the corresponding financial compensation.

\section{Participant timeline $\{13\}$}

A flowchart of the study design is illustrated in Fig 1. 


\section{Sample size $\{14\}$}

The sample size calculation is based on the clinical pregnancy rate. Studies indicated that clinical pregnancy rate of female infertility with RIF was $14.5 \%$ to $20.95 \%[19,20]$. We hypothesized that intrauterine administration of seminal plasma before FET can increase the clinical pregnancy rate from 17.5 to $35 \%$. According to the sample size of the estimation formula

$$
\mathrm{N}=(\mu \alpha+\mu \beta) 24 \bar{\pi}(1-\bar{\pi}) /(\pi 1-\pi 2) 2
$$

where $\mu \alpha=\mu 0.05=1.64, \mu \beta=\mu 0.8=0.84, \pi 1$ is the clinical pregnancy rate of the treatment group $=0.35, \pi 2$ is the clinical pregnancy rate of the control group $=0.175$, and $\bar{\pi}$ is the average of $\pi 1$ and $\pi 2 . \mathrm{N}=156$. To account for a $10 \%$ dropout rate, we will recruit 87 patients per arm.

\section{Recruitment $\{15\}$}

Methods of persuading the recruited subjects to participate in the trial: explain the advantages of the trial scheme and inform the patients that the cost of B-ultrasound in the endometrial preparation period is free of charge. However, care should be taken not to have any exaggerated or inconsistent words or assurances, so that normal recruitment is not persuasion.

\section{Assignment of interventions: allocation}

\section{Sequence generation $\{16 \mathrm{a}\}$}

Eligible participants will be randomly assigned to the experimental group (intrauterine administration of seminal plasma before FET) or the control group in a 1:1 ratio. Random numbers will be generated by using software SPSS 25.0 performed by an independent statistician from Chengdu Women's and Children's Central Hospital.

\section{Concealment mechanism $\{16 \mathrm{~b}\}$}

Opaque, sealed envelopes will be labelled with the randomisation number and will contain a sheet stating the group allocation for the patient. Randomisation envelopes will be used in consecutive order. Random numbers are unavailable to those who enrol participants or assign interventions. Study personnel are all blinded to the upcoming treatment group allocation. If patients are excluded from the study after randomisation, their numbers will not be reused.

\section{Implementation $\{16 c\}$}

Outpatient doctor who will enrol participants, and the main investigators will assign participants to interventions.

\section{Assignment of interventions: Blinding}

\section{Who will be blinded $\{17 \mathrm{a}\}$}


Only the data analyst will be blinded to group assignment. Operator of FET, attending physicians, nursing staff, and patients and families cannot be blinded in this study, as the procedures differ between groups; however, outcome assessors will be blinded.

\section{Procedure for unblinding if needed $\{17 \mathrm{~b}\}$}

Not applicable.

\section{Data collection and management}

\section{Plans for assessment and collection of outcomes $\{18 \mathrm{a}\}$}

All data will be collected using an CRF and will be supervised by an independent statistician from Chengdu Women's and Children's Central Hospital.

\section{Plans to promote participant retention and complete follow-up $\{18 \mathrm{~b}\}$}

Give the contact information of the project leader to the patient and keep in touch at any time Through telephone follow-up survey, and Chengdu Women's and Children's Central Hospital will be responsible for compensation to those who suffer harm from trial participation.

\section{Data management $\{19\}$}

All data will be collected using an CRF and will be supervised by an independent statistician from Chengdu Women's and Children's Central Hospital. Paper files will be kept in a locked filing cabinet in the department of reproduction and infertility. All research documents will be preserved for at least 5 years after publication. All patients signed up agreement document for follow-up during the trial. We can reach any patient through the community or women's federation in Sichuan

\section{Confidentiality $\{27\}$}

Paper files will be kept in a locked filing cabinet in the department of reproduction and infertility. All research documents will be preserved for at least 5 years after publication.

\section{Plans for collection, laboratory evaluation and storage of biological specimens for genetic or molecular analysis in this trial/future use $\{33\}$}

Not applicable.

\section{Statistical methods}




\section{Statistical methods for primary and secondary outcomes $\{20 \mathrm{a}\}$}

Statistical analysis will be performed using SPSS 25.0 (IBM Corp., Armonk, NY, USA). All data were entered into IBM SPSS Statistics 25. The statistical significance level was set at 0.05. Numerical variables were tested for their normality using the Kolmogorov-Smirnov test. Normally distributed variables are presented as the mean \pm standard error of the mean and were compared using ANOVA. Continuous variables with nonnormal distributions are expressed as the median and range and were compared using the nonparametric method (Kruskal-Wallis rank-sum test or Wilcoxon-Mann-Whitney test). Independent sample t-tests were used for continuous variables. Nominal variables are reported in the form of frequencies with percentages and were compared using the chi-square test or Fisher's exact test. The relationship between these factors and cumulative pregnancy rate/delivery of a healthy baby (baby-take home rate) was studied using conditional logistic regression, and the estimated odds ratios (ORs) and their 95\% confidence intervals (CIs) are reported. Intention-to-treat analysis will be applied to minimize bias due to dropouts, and reasons for which patients have dropped out will be recorded in detail and analyzed after the trial. Multiple imputation analysis will be used for missing data.

\section{Interim analyses $\{21 \mathrm{~b}\}$}

Since and education department of Chengdu Women's and Children's Central Hospital will be responsible for interim analyses and make the final decision to terminate the trial.

Methods for additional analyses (e.g. subgroup analyses) $\{20 \mathrm{~b}\}$

Not applicable.

\section{Methods in analysis to handle protocol non-adherence and any statistical methods to handle missing data $\{20 \mathrm{c}\}$}

Normally distributed variables are presented as the mean \pm standard error of the mean and were compared using ANOVA. Continuous variables with nonnormal distributions are expressed as the median and range and were compared using the nonparametric method (Kruskal-Wallis rank-sum test or Wilcoxon-Mann-Whitney test). Multiple imputation analysis will be used for missing data.

\section{Plans to give access to the full protocol, participant level-data and statistical code $\{31 \mathrm{c}\}$}

The protocol will be available via additional file. There are currently no plans to grant public access to the participant-level dataset or the statistical code.

\section{Oversight and monitoring}

\section{Composition of the coordinating centre and trial steering committee $\{5 \mathrm{~d}\}$}

Not applicable. 


\section{Composition of the data monitoring committee, its role and reporting structure $\{21 \mathrm{a}\}$}

Data monitoring committee (DMC): Ethics Committee of Chengdu Women's and Children's Central Hospital. It is independent from the sponsor and competing interests.

\section{Adverse event reporting and harms $\{22\}$}

During the study, serious adverse events will be immediately reported to the principal investigator, and appropriate measures will be initiated instantly.

The Ethics Committee will determine whether the adverse event is likely to have been associated with the experimental drug, and the sponsor will provide the cost of the treatment associated with the study and the corresponding financial compensation.

\section{Frequency and plans for auditing trial conduct \{23\}}

Ethics Committee of Chengdu Women's and Children's Central Hospital. It is independent from the sponsor and competing interests.

\section{Plans for communicating important protocol amendments to relevant parties (e.g. trial participants, ethical committees) $\{25\}$}

If there any important protocol amendments we have to report to Ethics Committee of Chengdu Women's and Children's Central Hospital.

\section{Dissemination plans $\{31 \mathrm{a}\}$}

We will report our results to Chinese clinical trial registry (chictr.org.cn). Fang Wang is responsible for approving the content and dissemination of all publications, abstracts and presentations arising from the trial and for assuring the confidentiality and integrity of the trial.

\section{Discussion}

RIF has been a great challenge in the field of reproductive medicine. We are looking for effective and direct treatments for RIF. There have some kind of treatments of RIF including immune therapy. However, the effectiveness of these treatments is uncertain. Successful embryo implantation is a dynamic process. In a natural mating situation, there is seminal plasma in the endometrium after mate before embryo implantation. The seminal plasma contains NGF, it can change the gene expressions associated with implantation[16]. This is a trial evaluating whether the intrauterine administration of seminal plasma before ET could improve clinical pregnancy rate in patients with RIF under the age of 39 years who are undergoing FET after IVF or ICSI or PGT-A.

In this study, the participants' age will be limited to 20 to 39 years in the study as age is recognized as an independent adverse factor for succuss of pregnancy, including the men and women. Men's semen quality was also correlated with age. Age-associated declines in semen volume, percentage 
motility, progressive motility, normal morphology and unfragmented cells were statistically significant[23].

The main limitation of the study is that it is not blinded. Blinding is not possible for reproductive specialists and participants. Because specialists perform the ET surgery and participants will know if the male don't provide semen.

There was not one RCT to assess the efficacy of intrauterine administration of seminal plasma before ET in the whole world. This RCT is very novel. On the basis of more than 10 years of IVF/ICSI practice, and more than 2000 cycles of IVF/ICSI/PGT cycles per year in our department. We have extensive experience in embryo transfer and intrauterine perfusion. This study is expected to provide a reliable way to improve clinical rate in patients with RIF.

\section{Trial status}

The date of approval is 31rd May 2021, version 2.0. Recruitment started on 1rd June and is expected to end in July 2022.

\section{Abbreviations}

\begin{tabular}{|l|l|}
\hline AE & Adverse event \\
\hline CRF & Case report form \\
\hline FET & Embryo transfer \\
\hline hCG & Frozen-thawed embryo transfer \\
\hline ICSI & Human chorionic gonadotrophin \\
\hline IVF & Intracytoplasmic sperm injection \\
\hline PAGT & In vitro fertilization \\
\hline RCT & Platelet aggregation test \\
\hline RIF & Randomized controlled trial \\
\hline SAE & Recurrent implantation failure \\
\hline
\end{tabular}

\section{Declarations}




\section{Acknowledgements}

The authors thank statistician from Chengdu Women's and Children's Central Hospital.

\section{Authors' contributions $\{31 \mathrm{~b}\}$}

Ling Cui is the Chief investigator, she conceived of the study, design of the study and performed the statistical analysis and drafted the manuscript. Fang Wang participated in its design and coordination and development of the proposal. Yonghong Lin participated in its design and coordination study. All authors read and approved the final manuscript.

\section{Funding $\{4\}$}

This work was supported by the Sichuan Medical Association grant (S18071) and Chengdu Women's and Children's Central Hospital.

\section{Availability of data and materials $\{29\}$}

The datasets generated during the current study are available from the corresponding author on reasonable request.

\section{Ethics approval and consent to participate $\{24\}$}

The study protocol has been approved by the ethics committees at Chengdu Women's and Children's Central Hospital, No. 2021(35). Written, informed consent to participate will be obtained from all participants"

\section{Consent for publication $\{32\}$}

Not applicable.

\section{Competing interests $\{28\}$}

The authors declare that they have no competing interests.

\section{Authors' information (optional)}

Ling Cui, Yonghong Lin, Fang Wang. Department of Reproduction and Infertility, Chengdu Women's and Children's Central Hospital, School of Medicine, University of Electronic Science and Technology of China, Chengdu 611731, China.Corresponding authors: Fang Wang, email: postwf@163.com 


\section{References}

1. Cao H, You D, Yuan M, Xi M (2018) Hysteroscopy after repeated implantation failure of assisted reproductive technology: A meta-analysis. J Obstet Gynaecol Res 44: 365-373.

2. Polanski LT, Baumgarten MN, Quenby S, Brosens J, Campbell BK, et al. (2014) What exactly do we mean by 'recurrent implantation failure'? A systematic review and opinion. Reprod Biomed Online 28: 409-423.

3. Coughlan C, Ledger W, Wang Q, Liu F, Demirol A, et al. (2014) Recurrent implantation failure: definition and management. Reprod Biomed Online 28: 14-38.

4. Macklon N (2017) Recurrent implantation failure is a pathology with a specific transcriptomic signature. Fertil Steril 108: 9-14.

5. Koot YEM, Hviid Saxtorph M, Goddijn M, de Bever S, Eijkemans MJC, et al. (2019) What is the prognosis for a live birth after unexplained recurrent implantation failure following IVF/ICSI? Hum Reprod 34: 2044-2052.

6. RoyChoudhury S, Singh A, Gupta NJ, Srivastava S, Joshi MV, et al. (2016) Repeated implantation failure versus repeated implantation success: discrimination at a metabolomic level. Hum Reprod 31: 1265-1274.

7. Pundir J, El Toukhy T (2010) Uterine cavity assessment prior to IVF. Womens Health (Lond) 6: 841-847; quiz 847848 .

8. Bozdag G, Aksan G, Esinler I, Yarali H (2008) What is the role of office hysteroscopy in women with failed IVF cycles? Reprod Biomed Online 17: 410-415.

9. Lass A, Peat D, Avery S, Brinsden P (1998) Histological evaluation of endometrium on the day of oocyte retrieval after gonadotrophin-releasing hormone agonist-follicle stimulating hormone ovulation induction for in-vitro fertilization. Hum Reprod 13: 3203-3205.

10. Karizbodagh MP, Rashidi B, Sahebkar A, Masoudifar A, Mirzaei H (2017) Implantation Window and Angiogenesis. J Cell Biochem 118: 4141-4151.

11. Shaulov T, Sierra S, Sylvestre C (2020) Recurrent implantation failure in IVF: A Canadian Fertility and Andrology Society Clinical Practice Guideline. Reprod Biomed Online 41: 819-833.

12. Yang DN, Wu JH, Geng L, Cao LJ, Zhang QJ, et al. (2020) Efficacy of intrauterine perfusion of peripheral blood mononuclear cells (PBMC) for infertile women before embryo transfer: meta-analysis. J Obstet Gynaecol 40: 961-968.

13. Bar G, Harlev A, Alfayumi-Zeadna S, Zeadna A, Bord I, et al. (2020) Recurrent implantation failure: which patients benefit from endometrial scratching prior to IVF? Arch Gynecol Obstet 301: 817-822.

14. Cozzolino M, Diaz-Gimeno P, Pellicer A, Garrido N (2020) Evaluation of the endometrial receptivity assay and the preimplantation genetic test for aneuploidy in overcoming recurrent implantation failure. J Assist Reprod Genet 37: 2989-2997.

15. Dieamant F, Vagnini LD, Petersen CG, Mauri AL, Renzi A, et al. (2019) New therapeutic protocol for improvement of endometrial receptivity (PRIMER) for patients with recurrent implantation failure (RIF) - A pilot study. JBRA Assist Reprod 23: 250-254.

16. Gardela J, Jauregi-Miguel A, Martinez CA, Rodriguez-Martinez H, Lopez-Bejar M, et al. (2020) Semen Modulates the Expression of NGF, ABHD2, VCAN, and CTEN in the Reproductive Tract of Female Rabbits. Genes (Basel) 11.

17. O'Leary S, Jasper MJ, Warnes GM, Armstrong DT, Robertson SA (2004) Seminal plasma regulates endometrial cytokine expression, leukocyte recruitment and embryo development in the pig. Reproduction 128: 237-247.

18. Robertson SA, O'Leary S, Armstrong DT (2006) Influence of semen on inflammatory modulators of embryo implantation. Soc Reprod Fertil Suppl 62: 231-245.

19. Kalem Z, Namli Kalem M, Bakirarar B, Kent E, Makrigiannakis A, et al. (2020) Intrauterine G-CSF Administration in Recurrent Implantation Failure (RIF): An Rct. Sci Rep 10: 5139.

20. Yu N, Zhang B, Xu M, Wang S, Liu R, et al. (2016) Intrauterine administration of autologous peripheral blood mononuclear cells (PBMCs) activated by HCG improves the implantation and pregnancy rates in patients with repeated implantation failure: a prospective randomized study. Am J Reprod Immunol 76: 212-216.

21. Alpha Scientists in Reproductive M, Embryology ESIGo (2011) The Istanbul consensus workshop on embryo assessment: proceedings of an expert meeting. Hum Reprod 26: 1270-1283.

22. Zegers-Hochschild F, Adamson GD, de Mouzon J, Ishihara O, Mansour R, et al. (2009) The International Committee for Monitoring Assisted Reproductive Technology (ICMART) and the World Health Organization (WHO) Revised Glossary on ART Terminology, 2009. Hum Reprod 24: 2683-2687.

23. Johnson SL, Dunleavy J, Gemmell NJ, Nakagawa S (2015) Consistent age-dependent declines in human semen quality: a systematic review and meta-analysis. Ageing Res Rev 19: 22-33. 
Figures

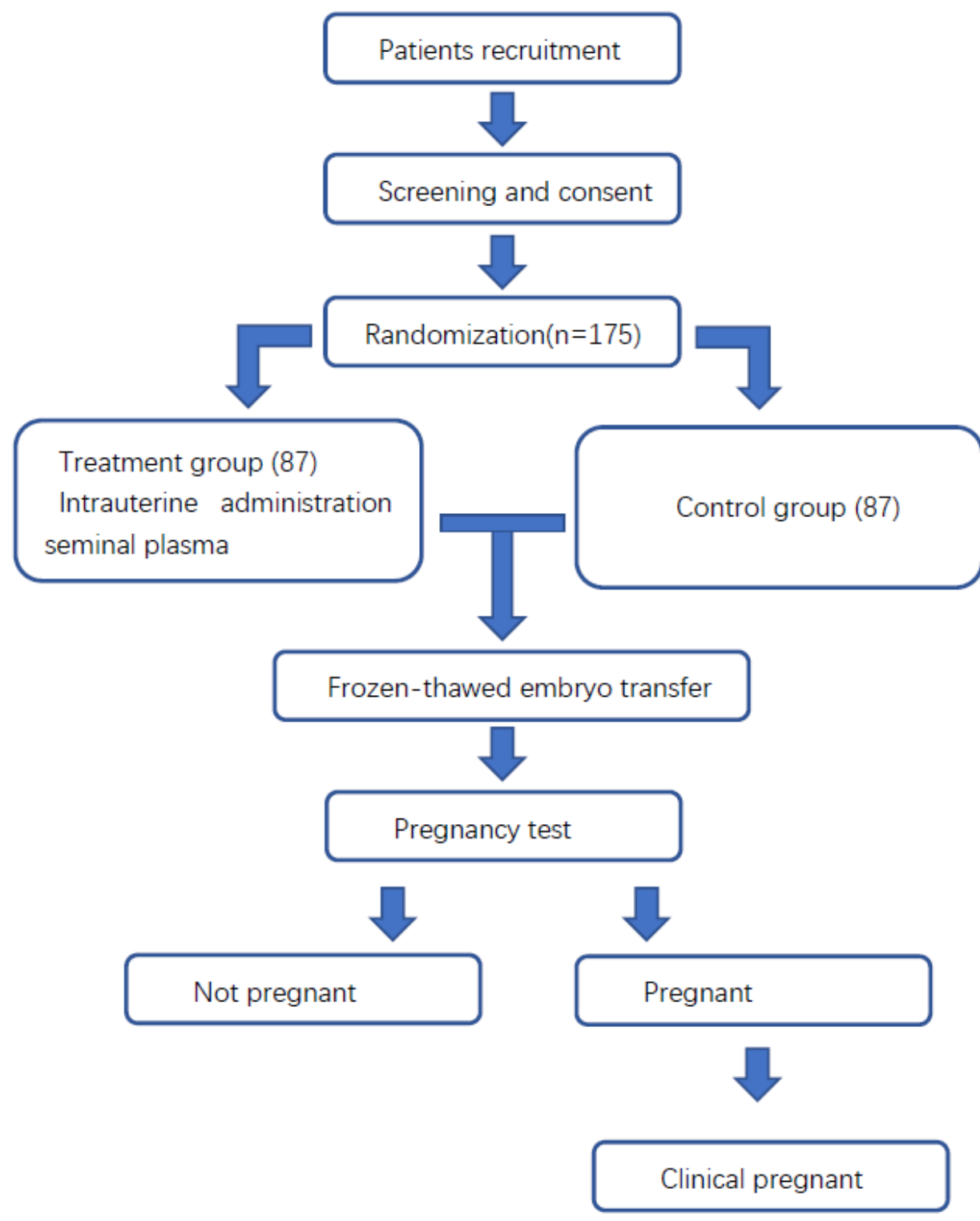

Figure 1 Flowchart of the study design

Figure 1

Please See image above for figure legend. 